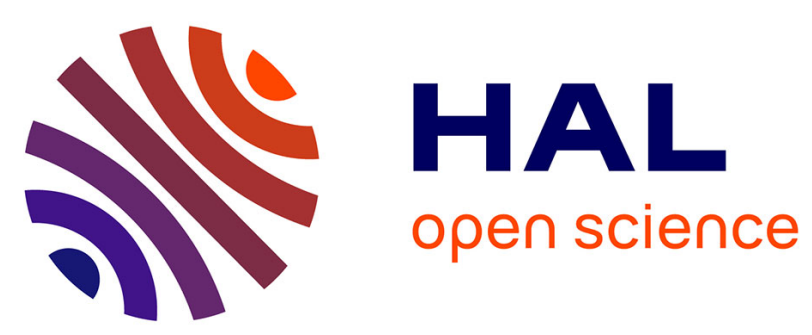

\title{
EXCITATION ACCOMPANYING PHOTOIONIZATION IN ATOMS AND MOLECULES AND ITS RELATIONSHIP TO ELECTRON CORRELATION
}

\author{
Tom Carlson, M. Krause, W. Moddeman
}

\section{To cite this version:}

Tom Carlson, M. Krause, W. Moddeman. EXCITATION ACCOMPANYING PHOTOIONIZATION IN ATOMS AND MOLECULES AND ITS RELATIONSHIP TO ELECTRON CORRELATION. Journal de Physique Colloques, 1971, 32 (C4), pp.C4-76-C4-84. 10.1051/jphyscol:1971416 . jpa00214617

\section{HAL Id: jpa-00214617 https://hal.science/jpa-00214617}

Submitted on 1 Jan 1971

HAL is a multi-disciplinary open access archive for the deposit and dissemination of scientific research documents, whether they are published or not. The documents may come from teaching and research institutions in France or abroad, or from public or private research centers.
L'archive ouverte pluridisciplinaire HAL, est destinée au dépôt et à la diffusion de documents scientifiques de niveau recherche, publiés ou non, émanant des établissements d'enseignement et de recherche français ou étrangers, des laboratoires publics ou privés. 


\title{
EXCITATION ACCOMPANYING PHOTOIONIZATION IN ATOMS AND MOLECULES AND ITS RELATIONSHIP TO ELECTRON CORRELATION
}

\author{
T. A. CARLSON, M. O. KRAUSE, and W. E. MODDEMAN (*) \\ Oak Ridge National Laboratory Oak Ridge, Tennessee, U. S. A.
}

\begin{abstract}
Résumé. - Si on néglige la corrélation électronique, un seul électron est émis par l'effet photoélectrique. Mais l'expérience montre qu'il existe une grande probabilité pour l'excitation ou l'ionisation simultanée d'un second électron. Cette excitation additionnelle est expliquée par les corrélations électroniques : de façon implicite dans les calculs de shakeoff électronique, avec des fonction d'ondes d'un seul électron, ou de façon explicite par l'utilisation des fonctions d'ondes corrélées. Nous avons étudié les pics satellites dans les spectres photoélectroniques des couches internes et externes des gaz rares et de quelques molécules simples. Ces pics satellites sont interprétés à partir des transitions discrètes de l'atome simplement ionisé. Elles nous permettent de mieux comprendre l'excitation simultanée dans l'émission photoélectrique.
\end{abstract}

\begin{abstract}
If electron correlation is neglected, the photoelectric effect requires that only one electron be ejected from an atom. Experimentally, however, it is found in the photoionization process that there is a high probability for simultaneous excitation or ionization of a second electron in the same atom. This extra excitation can be explained by electron correlation : in some cases implicitly through the use of single-electron wave functions as in electron shake-off calculations, and in other cases through the use of wave functions that explicitly include correlation. We have utilized two recently constructed high resolution electron spectrometers to measure satellite lines in the photoelectron spectra of both the inner and valence shells of the rare gases and some simple gaseous molecules. These satellite lines are interpreted in terms of transitions to excited states of the singly charged ion, and are used to form a broader basis for the understanding of simultaneous excitation as the result of the photoelectron process.
\end{abstract}

I. Introduction. - In principal the photoelectric effect involves the ejection of only one electron. Experimentally [1-8], however, multiple ionization occurs in the photoelectron process with a probability of the order of $20 \%$. This apparent paradox is easily understood when the effects of electron correlation are considered. These effects can be divided into two categories. The first case occurs when an electron in the outer shell of an atom feels a sudden change in the central potential as the result of the photoejection of an inner shell electron. Here the effect of electron correlation is simply the change in electron shielding. In normal parlance this is generally not even spoken of as electron correlation. The second category involves the ejection of two electrons or more from the same principal shell. In this situation it is necessary when accounting for the probability of this occurrence to use wave functions that explicitly include electron correlation.

To explain the experimental data on multiple ioni-

(*) Research sponsored by the U. S. Atomic Energy Commission under contract with the Union Carbide Corporation.

(*) Graduate student from the University of Tennessee supported by a National Aeronautics Research Grant; present address : Vanderbilt University, Nashville, Tennessee. zation occurring in the photoelectron process the sudden approximation was invoked [1-9] which states that if the Hamiltonian changes suddenly the transition probability for going to a given final state can be evaluated in terms of the square of the overlap integral between the initial and final states. For the specific case of photoionization in an inner shell the probability for simultaneously exciting or ejecting an electron from an outer orbital $n l j$ is

$$
P_{n 1 \mathrm{j} \rightarrow n^{\prime} \mathrm{lj}}=1-\left|\int \psi_{n 1 \mathrm{j}}^{*} \psi_{n 1 \mathrm{j}}^{\prime} \mathrm{d} \tau\right|^{2 N}
$$

where $\psi_{n 1 j}$ is the single-electron wave function for the orbital $n$ lj in ground state of the neutral atom and $\psi_{n \mathrm{j}}^{\prime}$ is the single-electron wave function corresponding to the same orbital but for an ion containing an inner shell vacancy. With the use of Hartree-Fock wave functions good agreement was obtained between theory and experiment. The process is similar to autoionization observed in beta decay [9] except that whereas in beta decay the change in the central potential is due to a sudden change in nuclear charge, in photoionization the change is due to the removal of an electron which was shielding the charge. The 
method of the sudden approximation has been further extended to the explanation of X-ray satellite lines $[10,11]$.

When photoionization takes place in the outer shell, electrons from the saine shell can also be ejected. When the probability for this was calculated with single-electron-wave functions by means of an expression similar to eq. (1), it was found that the experimental values were much larger than those calculated [4]. We reasoned that the discrepancy arose because multiple electron ejection from a shell having the same principal quantum number required that electron correlation be considered explicitly. Indeed Byron and Joachain [12] showed by using electron correlation explicitly in the initial bound state of helium that the experimental data on double ionization could be successfully accounted for. Áberg [13] demonstrated that the sudden approximation was also valid in the situation when two electrons were removed from the same shell if again correlation is included explicitly. When a photoelectron emerges, it has a kinetic energy which depends on the final state of the ion. If extra excitation or ionization has occurred as the result of the photoelectron ejection, this will be reflected as a loss in energy for the photoelectron. Thus a study of the photoelectron spectrum is a study of the ionization and excitation processes that accompany the photoionization. While our earlier experimental work pertained mainly to the simultaneous transition of a second electron to the continuum state as the result of photoionization, some limited data $[4,6]$ on transitions to excited but discrete states were also obtained. Our earlier studies were done on an electron spectrometer which had the characteristics of low background but only modest resolution, suitable for continuum states but limited for measuring discrete lines. Our present spectrometers have energy resolution capability of better than $0.05 \%$ FWHM and are well equiped for studying discrete states: In this paper we shall present a variety of results obtained with our new instruments on both atomic and molecular systems together with comparison with theoretical predictions. We shall also include for comparison some excellent results of a similar nature recently reported by Siegbahn et al. [14].

II. Experiment. — Our photoelectron spectra were obtained with double focus electrostatic electron spectrometers. Most of our data were taken with an instrument containing two spherical sectors having a mean radius of $20 \mathrm{~cm}$. The design and operation of this spectrometer has been previously discussed elsewhere [15]. Some data were also obtained with a machine of similar design but slightly smaller radius $(15 \mathrm{~cm})$.

The principal purpose of the experiment was to measure the kinetic energy of photoelectrons ejected when a gas target was subjected to irradiation by characteristic X-rays, mainly, the $K_{\alpha_{1,2}}$ lines of $\mathrm{Mg}$ and Al. The kinetic energy of the photoelectron is given by

$$
E=h v-E_{\mathbf{B}}-E^{*}
$$

where $h v$ is the photon energy, $E_{\mathrm{B}}$ is the binding energy of an electron in a given orbital and $E^{*}$ is the energy arising from monopole excitation or ionization (*). The most intense line is observed when $E^{*}=0$. The lower energy satellite lines indicate the energy required and the probability for excitation.

The weighted average of X-ray energies [16] for the $K_{\alpha_{1}, 2}$ lines of $\mathrm{Al}$ and $\mathrm{Mg}$ are respectively $1486.6 \mathrm{eV}$ and $1253.6 \mathrm{eV}$ and their natural widths approximately $1 \mathrm{eV}$. The spectrometers were normally run with a resolution of $0.09 \%$ FWHM.

To determine the importance of collision losses in our experiments, we made runs at several pressures (the lines due to monopole excitation should be pressure independent while those that arise from colljsions suffered by the electron as they traverse the spectrometer are pressure dependent). The normal pressure was $\sim 10$ microns in the source volume and $10^{-5}$ torr in the spectrometer. Separate experiments using an electron gun were also used to determine the nature and extent of the collision lines. Intensities were usually compared using areas under the peak rather than peak heights and were corrected for energy window widths.

III. Monopole excitation as the result of an inner shell vacancy. - Neon offers an ideal case for studying the effects of photoionization in the inner shell, since its ten electrons fill both the $K$ and $L$ shells, and the $1 \mathrm{~s}$ electrons can be ejected with $\mathrm{Al} K_{\alpha}$ or $\mathrm{Mg} K_{\alpha}$ X-rays. The photoelectron spectrum has been previously determined by ourselves [6] but with an interest aimed primarily towards measuring transitions to the continuum state. The resolution in the earlier studies was insufficient for correctly determining the effects of monopole excitation. We have repeated these experiments using both $\mathrm{Al}$ and $\mathrm{Mg}$ $\mathrm{X}$-rays and the results are listed in Table I. The spectrum using $\mathrm{Mg} K_{\alpha}$ lines is given in figure 1 . The main peak is due to the photoejection of a $1 \mathrm{~s}$ electron without excitation by a $\mathrm{Mg} K_{\alpha_{1,2}}$ X-ray. Its kinetic energy is $383.4 \mathrm{eV}$ from eq. (2) where the binding energy is taken [14] as $870.2 \mathrm{eV}$. We have related all other energies to this peak. The dotted line in figure 1 gives the spectra due to inelastic losses expected from the main peak. The net spectrum contains small contributions from the X-ray satellites of $\mathrm{Mg}: K_{\alpha^{\prime}}$, $K_{\alpha_{3}}, K_{\alpha_{4}}$ and $K_{\alpha_{5,6}}$ which appear to the high energy

(*) The operator in eq. (1) is unity and transitions to the final state obey the rule for monopole interaction. We thus will use the term monopole ionization when we refer to processes where electrons are put into the continuum and monopole excitation when the transitions are to describe excited states. The processes have also been called [14] respectively electron shake-off and electron shake-up. 


\section{TABLE I}

Photoelectron Spectrum of Neon Ionized in the $K$ Shell (Monopole excited states)

Designation

$1 \mathrm{~s} 2 \mathrm{~s}^{2} 2 \mathrm{p}^{6} 2 \mathrm{~S}$

$1 s 2 s^{2} 2 p^{5} 3 p{ }^{2} s$ lower

$1 \mathrm{~s} 2 \mathrm{~s}^{2} 2 \mathrm{p}^{5} 3 \mathrm{p}$ 2S upper

$1 \mathrm{~s} 2 \mathrm{~s}^{2} 2 \mathrm{p}^{5} 4 \mathrm{p}$ 2s lower

$1 \mathrm{~s} 2 \mathrm{~s}^{2} 2 \mathrm{p}^{5} 5 \mathrm{p}^{2} \mathrm{~S}$ lower

$1 \mathrm{~s} 2 \mathrm{~s}^{2} 2 \mathrm{p}^{5} 4 \mathrm{p} 2 \mathrm{~S}$ upper

$1 \mathrm{~s} 2 \mathrm{~s}^{2} 2 \mathrm{p}^{5} \mathrm{np}$ (remainder)

Ionization limit

$1 \mathrm{~s} 2 \mathrm{~s} 2 \mathrm{p}^{6} 3 \mathrm{~s}$

$1 \mathrm{~s} 2 \mathrm{~s} 2 \mathrm{p}^{6} 4 \mathrm{~s}$
Energy (eV relative to unexcited transition)

\begin{tabular}{ccc}
$\operatorname{Run} A(a)$ & $\operatorname{Run~B(b)}$ & \multicolumn{1}{c}{$\left(^{(c)}\right.$} \\
$\frac{-}{0}$ & $\overline{0}$ & $\overline{0}$ \\
$37.3(2)$ & $37.1(3)$ & 37.3 \\
$40.7(2)$ & $40.5(3)$ & 40.7 \\
$42.4(2)$ & $42.0(3)$ & 42.3 \\
$44.4(2)$ & - & 44.2 \\
- & - & 46.4 \\
$\sim 46$ & $\sim 46$ & - \\
$\sim 47.4$ & $\sim 48$ & $\sim 47$ \\
$59.5(5)$ & $59.1(7)$ & 60 \\
$68(2)$ & - & -
\end{tabular}

Intensity (relative to unexcited transition)

Theory ${ }^{(d)}$
0(868.6)
35.6
39.5
40.5
42.4
44.6
-
45.2
61

(a) $\mathrm{Mg} K_{\alpha} \mathrm{X}$-rays used.

(b) Al $K_{\alpha}$ X-rays used.

(c) Cf. Ref. 14 .

(d) Energies were obtained from Hartree-Fock wave functions employing Slater corrections for unfilled shells (cf. Ref. 14).

(e) Intensities were calculated using equation 3 (cf. Ref. 6).

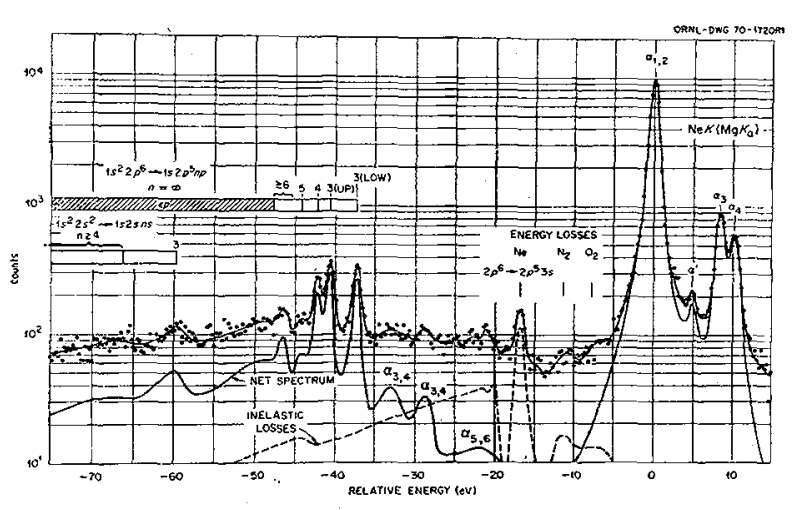

Fig. 1. - Photoelectron Spectrum of Neon Ionized in the $K$ Shell by $\operatorname{Mg} K_{\alpha}$ X-rays. Energy given relative to principal peak whose kinetic energy is $383 \mathrm{eV}$. Dotted line shows inelastic losses as determined in separate experiments. Net spectrum is derived from substracting the background and contribution from inelastic scattering from the gross data.

side of the principal line arising from $K_{\alpha_{1,2}}$. The three lines of most interest to this study occur at $37.3,40.7$, and $42.3 \mathrm{eV}$ and are the principal contributions of the monopole excitation process. In each case an electron in the $2 \mathrm{p}$ orbital is excited into a higher principal quantum number. According to monopole selection rules there should be no change in angular momentum or spin. The upper and lower states arise according to whether the spin of the excited electron is parallel or antiparallel to that of the remaining electrons in the $1 \mathrm{~s}$ orbital. Bagus and Gelius [17] calculated Hartree-Fock atomic wave functions corrected by Slater coefficients for $L S$ coupling and minimized to the lowest total energy. The energies taken from these solutions are given in Table I. Also given in Table I are the experimental results of Siegbahn et al. [14]. The agreement with ours is in general quite satisfactory. The agreement between experimental energies and calculated ones are reaso- nably good and the assignment of the states seem dependable.

Also available for comparison are the intensities of the lines due to monopole excitation. The transition probability is given as

$$
P_{n^{\prime} \mathbf{l j}}=N\left|\int \psi_{n ! \mathrm{j}} \psi_{n^{\prime} 1 \mathrm{j}} \mathrm{d} \tau\right|^{2}
$$

where $P_{n^{\prime} \mid \mathrm{j}}$ is the probability an electron in the orbital $n \mathrm{lj}$ will be excited to the $n$ 'lj orbital as the result of an inner shell vacancy. $N$ is the number of electrons in the $n l j$ orbital. If we can distinguish between the final spin states, the number should be $N / 2$. Single electron wave functions from Hartree-Fock solutions similar to those described above for obtaining the energies were used [18], $\psi_{n 1 j}$ corresponding to the orbital $n \mathbf{l j}$ in the ground state of the atom, and $\psi_{n^{\prime} 1 j}$ corresponding to the orbital of the excited state of the singly charged ion having the appropriate configuration as given in Table $\mathrm{I}$. The agreement between experiment and theory is quite encouraging.

In summary, the effects of a sudden formation of an inner shell vacancy can be satisfactorily explained in terms of the sudden approximation using singleelectron wave functions.

IV. Monopole excitation as the result of photoionization in the outermost shell. - Photoionization in the outermost principal shell can also promote another electron of the same shell into a continuum or discrete state. Helium with its two $1 \mathrm{~s}$ electrons offers the simplest case for study. Earlier work on double ionization [4] showed that the probability for such an event was many times larger than that predicted from eq. (1) using single-electron wave functions. Similar conclusions were reached for neon and argon. We reasoned the discrepancy lay in the fact 
that single-electron wave functions were no longer adequate when the two electrons involved in the ionization were from the same shell. The wave functions would require an explicite inclusion of electron correlation. Byron and Joachain [12] showed that this was indeed the case by using a Hylleraas-type wave function for the ground state of $\mathrm{He}$ and thereby obtajning excellent agreement with our experimental data on double ionization. Åberg [13] has also shown that the concept of the sudden approximation is still valid when two electrons are removed from the same shell so long as electron correlation is explicitly included in the wave function for the initial state.

Some data on monopole excitation as the result of photoionization have been obtained by Carlson [4] at a photon energy of $278 \mathrm{eV}$ and by Samson [19] at $66.6 \mathrm{eV}$. In this paper we present data using the $1487 \mathrm{eV} K_{\alpha_{1,2}}$ line of Al. This give us an opportunity to study process as a function of energy. The sudden approximation requires that the excitation be independent of the details of the process causing the change in the potential, in this case the energy of the ejected photoelectron. Studies $[3,20]$ have been made for cases of monopole ionization and it was found that the ratio of single to double ionization was consistent over a wide energy range except near the threshold for double electron ejection where the probability dropped sharply towards zero. In the case of a more highly correlated system (such as when both electrons are in the same shell) Åberg $[13,21]$ suggests that asymptotic behavior is not rea hed until a much higher energy.

Figure 2 shows the photoelectron spectrum of $\mathrm{He}$ using the $\mathrm{Al} K_{\alpha_{1,2}}$ line. Two lines are discernible at -40.9 and $-48.5 \mathrm{eV}$ with respect to the main photo peak. These lines are in excellent agreement with optical data [22] for the $n=2$ and $n=3$ states of $\mathrm{He}^{+}$of -40.8 and $-48.4 \mathrm{eV}$. Since the $\mathrm{s}$ and $\mathrm{p}$

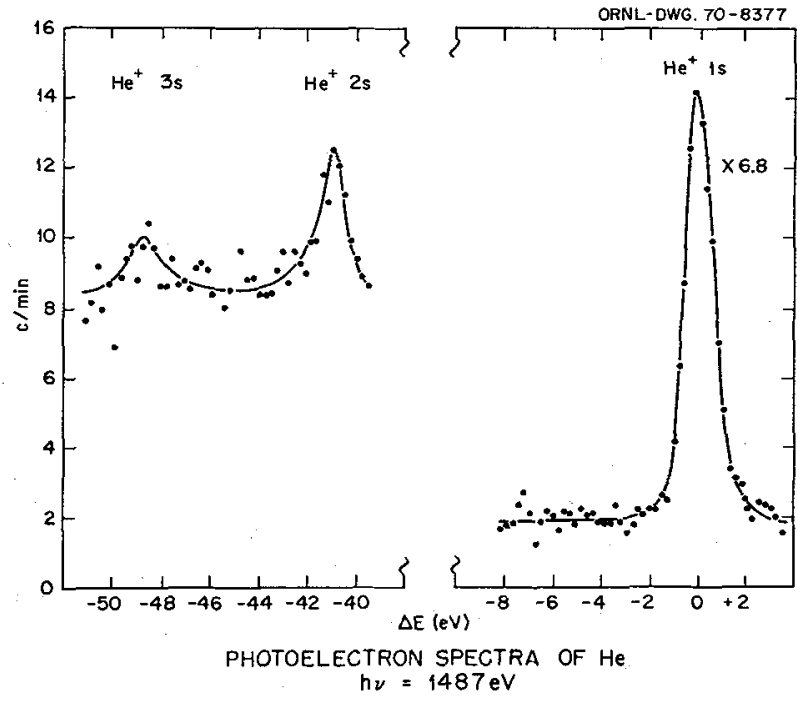

FIG, 2. - Photoelectron spectrum of helium ionized in the $K$ shell by $\mathrm{Al} K_{\alpha} \mathrm{X}$-rays. Energy given relative to principal peak whose kinetic energy is $1462 \mathrm{eV}$.

states are degenerate there is no way to tell from energy alone (at least in the limits of our resolution) the angular momentum of the final states. However, Brown [23] has examined this problem theoretically and concludes that the probability for exciting an electron into an $s$ state is two orders of magnitude more probable than for a $p$ state ; that is, transitions from the ground to excited states are indeed essentially monopole.

In Table II we have given transition probabilities to the first two excited states in $\mathrm{He}^{+}$as the result of photoionization. Experimental values are now available for 3 widely different photon energies. The agreement with theory based on a correlated wave function for the He atom is quite good. Also our recent value at a photon energy of $1487 \mathrm{eV}$ agrees well with the asymptotic value of Åberg, based on a

TABLE II

Relative Transition Probabilities to States of $\mathrm{He}^{+}$as the Result of Photoionization

\begin{tabular}{|c|c|c|c|c|c|c|c|c|}
\hline \multirow[b]{2}{*}{ State/Energy } & \multicolumn{3}{|c|}{ Exp } & \multicolumn{5}{|c|}{ Theory } \\
\hline & $67 \mathrm{eV}\left({ }^{a}\right)$ & $278\left(^{b}\right)$ & $1487\left(^{c}\right)$ & $67\left(^{d}\right)$ & $278\left(^{d}\right)$ & $1487\left(^{d}\right)$ & $\infty\left(^{e}\right)$ & $\infty\left(^{f}\right)$ \\
\hline $1 \mathrm{~s}$ & 100 & 100 & 100 & 100 & 100 & 100 & 100 & 100 \\
\hline $2 \mathrm{~s}$ & $9 \pm 2$ & $6 \pm 1$ & $5.0 \pm 0.8$ & $7.7(9.0)\left({ }^{g}\right)$ & $6.4(6.5)\left({ }^{g}\right)$ & $5.3(5.0)\left(^{g}\right)$ & 4.8 & 1.8 \\
\hline $3 \mathrm{~s}$ & 一 & - & $1.4 \pm 0.8$ & - & - & 0.9 & 一 & 5 \\
\hline $2 \mathrm{~s}$ & - & $3 \pm 1$ & 一 & 一 & 1.7 & - & 1.1 & 4 \\
\hline
\end{tabular}

$\left({ }^{a}\right)$ SAmson (J. A. R.), Phys. Rev. Letters, 1969, 22, 693.

(b) CARLSON (T. A.), Phys. Rev., 1967, 56, 142.

(c) This paper.

( $\left.{ }^{(}\right)$Brown (R. L.), Phys. Rev. A., 1970, 1, 341. Correlated wave functions are used.

$\left({ }^{e}\right)$ ÅBerg (T.), Phys. Rev. 1970, A2, 1726. Asymptotic limit using the sudden approximation with initial wave functions including correlation explicitly.

$(f)$ Use of single electron wave function from GrEen (L. C.) et al., Phys. Rev., 1959, 33, 757, in equation 1 (cf. Ref. 4).

$\left({ }^{g}\right)$ Salpeter (E. E.) and Zadir (M. H.), Phys. Rev., 1962, 125, 248. Correlated wave functions are used. 
correlated wave function. The value based on singleelectron wave functions is wholly inadequate.

Figures 3 and 4 give the photoelectron spectrum of neon and argon ionization in the outer shell using the Al $K_{\alpha}$ lines. Tables III and IV give the energies,

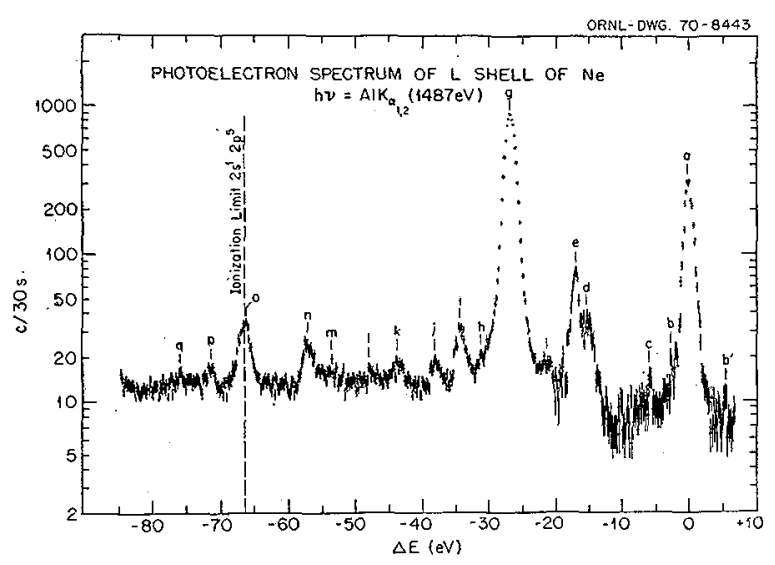

Fig. 3. - Photoelectron spectrum of neon ionized in the $L$ shell by $\mathrm{Al} K_{\alpha} \mathrm{X}$-rays. Energy given relative to first principal peak whose kinetic energy is $1465 \mathrm{eV}$. Identification of peaks is given in Table III. Ionization limits are the minimum energies needed to obtain the indicated configuration as referred to the ground state of $\mathrm{Ne}^{+} 2 \mathrm{~s}^{2} 2 \mathrm{p}^{5}$. An error bar is given for each data point from the square root of the total counts.

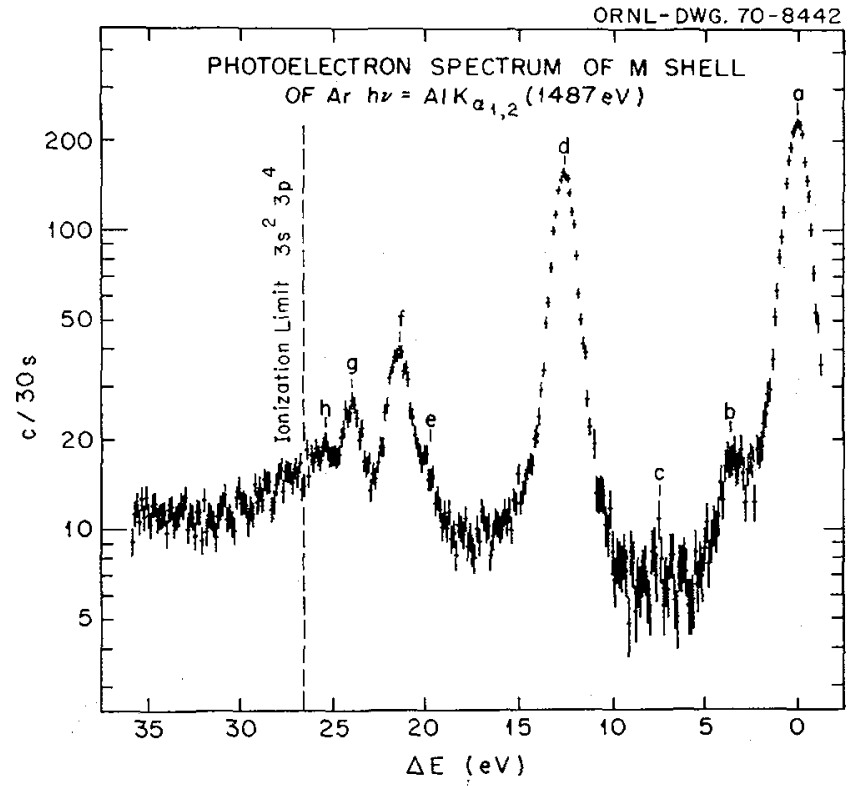

FIG. 4. - Photoelectron spectrum of argon ionized in the $M$ shell by $\mathrm{Al} K_{\alpha} \mathrm{X}$-rays. Energy given relative to first principal peak whose kinetic energy is $1471 \mathrm{eV}$. Identification of peaks is given in Table IV. Ionization limit is the minimum energy needed to reach the indicated configuration as referred to the ground state of $\mathrm{Ar}^{+} 3 \mathrm{~s}^{2} 3 \mathrm{p}^{5}$. An error bar is given for each data point from the square root of the total counts.

TABLE III

Analysis of Photoelectron Spectrum of Outermost Shell $(L)$ of $\mathrm{Ne} h v=\mathrm{Al}_{\alpha_{1,2}}(1487 \mathrm{eV})$

$\begin{array}{lcc}\text { Line } & \text { Energy }(\mathrm{eV})\left(^{1}\right) & \text { Intensity }\left(^{1}\right) \\ \overline{\mathrm{b}^{\prime}} & -\overline{5.3} & - \\ \mathrm{a} & 0 & 2 \\ \mathrm{~b} & 2.6 & 100 \\ \mathrm{c} & 6.0 & 1 \\ \mathrm{~d} & 15.3 & 1 \\ \mathrm{e} & 17.0 & 20 \\ \mathrm{f} & 21.1 & 1 \\ \mathrm{~g} & 26.7 & 273 \\ \mathrm{~h}(*) & 30.9 & 3 \\ \mathrm{i}\left(^{*}\right) & 34.0 & 6 \\ \mathrm{j}\left(^{*}\right) & 38.0 & 2 \\ \mathrm{k} & 43.4 & 2 \\ \mathrm{l} & 47.6 & 1 \\ \mathrm{~m} & 53.4 & 1 \\ \mathrm{n}\left(^{*}\right) & 57.1 & 4 \\ \mathrm{o}\left({ }^{*}\right) & 66.3\left(^{4}\right) & 7 \\ \mathrm{p} & 71.4 & 2 \\ \mathrm{q} & 75.8 & 1\end{array}$

Designation $\left({ }^{2}\right)$

$K_{\alpha^{\prime}}\left(2 \overline{\mathrm{s}^{2}} 2 \mathrm{p}^{5}\right)$

$2 \mathrm{~s}^{2} 2 \mathrm{p}^{5} \mathrm{p}^{0}$

$K_{\alpha 6}\left(2 \mathrm{~s}^{1} 2 \mathrm{p}^{6}\right)$

$K_{\alpha^{5}}\left(2 \mathrm{~s}^{1} 2 \mathrm{p}^{6}\right)$

$K_{\alpha^{4}}\left(2 \mathrm{~s}^{1} 2 \mathrm{p}^{6}\right)$

$K_{\alpha^{3}}\left(2 \mathrm{~s}^{1} 2 \mathrm{p}^{6}\right)$

$K_{\alpha^{\prime}}\left(2 \mathrm{~s}^{1} 2 \mathrm{p}^{6}\right)$

$2 \mathrm{~s}^{1} 2 \mathrm{p}^{62} \mathrm{~S}$

$2 \mathrm{~s}^{2} 2 \mathrm{p}^{4} 3 \mathrm{p}^{1}\left(3 \mathrm{p}^{2} \mathrm{p}^{0}\right)$

$2 s^{2} 2 p^{4} 3 p^{1}\left(3 p^{\prime 2} p^{0}\right)$

$2 \mathrm{~s}^{2} 2 \mathrm{p}^{4} 3 \mathrm{p}^{1}\left(3 \mathrm{p}^{\prime \prime} \mathrm{P}^{0}\right)$

main collision peak related to line $\mathrm{g}$

$$
\begin{gathered}
2 s^{1} 2 p^{5} 3 p^{1}{ }^{2} S \text { (lower) } \\
2 s^{1} 2 p^{5} 3 p^{1} S \text { (upper) }
\end{gathered}
$$

Energy

(Calculated) $\left({ }^{3}\right)$

$-\overline{5.5}$

3.6

7.1

15.4

17.4

21.4

26.9

31.5

34.3

37.9

43.5

(*) Lines designated as due to monopole excitation.

( $\left.{ }^{(}\right)$Experimental data (cf. Fig. 3) relative to first principal photo-electron peak (line a).

$\left({ }^{2}\right)$ Configuration and term value of final state. When photoelectron peaks arise from Al X-rays other than $K_{\alpha_{1,2}}$ they are so designated.

(3) Taken from optical data : Moore (C. E.), «Atomic Energy Levels», vol. I (NBS 467, U. S. Govt. Printing Office, Washington, D. C., 1949). For information on X-ray satellites of Al see Baun (W. L.) and Fisher (D. W.), Phys. Letters, 1964, 13, 36. The value in parenthesis is the first ionization potential for neon.

${ }^{(4)}$ May also contain slight contribution from W X-ray $M_{\mathrm{IV}} N_{\mathrm{III}}$ producing photo peak corresponding to $2 s^{1} 2 p^{6}$ final state of $\mathrm{Ne}$ (expected energy $\sim 67 \mathrm{eV}$ ). 
TABLE IV

\begin{tabular}{|c|c|c|c|c|}
\hline Line & Energy $\left({ }^{1}\right)(e V)$ & Intensity $\left({ }^{1}\right)$ & Designation $\left({ }^{2}\right)$ & $\begin{array}{c}\text { Energy } \\
\text { (Calculated) } \\
\left({ }^{3}\right)\end{array}$ \\
\hline- & $\frac{1}{0}$ & $\overline{100}$ & $3 \mathrm{~s}^{2} \cdot \overline{\mathrm{n}^{5}}{ }^{2} \mathrm{p}^{0}$ & $0(\overline{15.76})$ \\
\hline $\begin{array}{l}\mathrm{a} \\
\mathrm{b}\end{array}$ & 3.8 & $\begin{array}{r}100 \\
4\end{array}$ & $K_{0}\left(3 \mathrm{~s}^{1} 3 \mathrm{p}^{6}\right)$ & $\begin{array}{c}0(15.10) \\
4.0\end{array}$ \\
\hline c & 7.9 & 1 & $K$ & 8.0 \\
\hline d & 13.4 & 69 & $3 s^{1} 3 p^{6}$ & 13.5 \\
\hline e (*) & 20.8 & 2 & $3 s^{2} 3 p^{4}\left({ }^{3} P\right) 4 p^{1}\left(4 p^{2} p^{0}\right)$ & 19.8 \\
\hline $\mathrm{f}(*)$ & 22.6 & 13 & $3 s^{2} 3 p^{4}\left({ }^{1} S\right) 4 p^{1}\left(4 p^{\prime 2} P^{0}\right)$ & 21.4 \\
\hline$g(*)$ & 24.8 & 6 & $3 s^{2} 3 p^{4}\left({ }^{3} P\right) 4 p^{1}\left(4 p^{\prime \prime 2} p^{0}\right)$ & 23.8 \\
\hline $\mathrm{h}$ & 26.3 & 2 & - & - \\
\hline
\end{tabular}

(*) Lines designated as due to monopole excited states.

(1) Experimental data (cf. Fig. 4) relative to first principal photoelectron peak (line a).

$\left({ }^{2}\right)$ Configuration and term value of final state. When photoelectron peak arise from Al X-rays other than $K_{\alpha_{1,2}}$ they are so designated.

$\left({ }^{(3)}\right)$ Taken from optical data : Moore (C. E.), «Atomic Energy Levels », vol. I (NBS 467, U. S. Govt. Printing Office, Washington, D. C., 1949). For information on X-ray satellites of Al see Baun (W. L.) and Fisher (D. W.), Phys. Letters, 1964, 13, 36. The value in parenthesis is the first ionization potential for neon.

intensities and assignment of the lines observed.

In neon we find a trio of lines $(h, i$ and $j)$ that correspond very closely to monopole excited states arising from photoionization in the $2 \mathrm{p}$ shell. We have also assigned the peaks $\mathrm{n}$ and o to monopole excited states arising from photoionization in the $2 \mathrm{~s}$ shell. Optical data do not exist for these lines, but if we consider the electron in the $3 \mathrm{p}$ level as a spectator electron not strongly involved in the correlation, the splitting of the two lines should be approximately that found for the $2 \mathrm{~s} 2 \mathrm{P}^{5}{ }^{3} \mathrm{P}$ and ${ }^{1} \mathrm{P}$ states of $\mathrm{Ne}$ III which is $10.6 \mathrm{eV}$. This separation fits reasonably the observed splitting between lines $\mathrm{n}$ and $\mathrm{o}$ of $9.2 \mathrm{eV}$. Line o could also contain some contamination from the tungsten X-ray $M_{\mathrm{rV}}-N_{\mathrm{III}}$ whose energy is $1446 \mathrm{eV}$. However, the absence of other lines that would be produced by this X-ray leads us to set an upper limit of contamination of approximately $1 / 3$ the observed intensity.

The data on argon also show a trio of lines (e, $f$ and $\mathrm{g}$ ) corresponding to monopole excitation as the result of photoionization in the $3 \mathrm{p}$ orbital. If the excited orbital was $4 \mathrm{~s}$ rather than $4 \mathrm{p}$ indicating a dipole transition, the resulting states would occur in the energy range 16.6 to $20.7 \mathrm{eV}$. The absence of strong lines in this region further strengthens the feeling we are dealing primarily with monopole excitation. No strong lines corresponding to monopole excitation as the result of photoionization in the $3 \mathrm{~s}$ shell were seen.

The probability for monopole excitation as the result of a vacancy in the same shell appears to be the same order of magnitude as when an inner shell vacancy is formed. Single-electron wave functions would predict a much lower value, but, as demonstrated in the case of He electrons, this is counterbalanced by the inclusion of electron correlation.
In summary, monopole excitation can occur when photoionization takes place in the outer shell, but unlike the case when a vacancy is in an inner shell the probability cannot be correctly calculated using single-electron wave functions. But by using correlation explicitly in the initial bound states, as in the case of $\mathrm{He}$, the transition probabilities can be obtained in fairly good agreement with experiment.

V. Monopole excitation in molecules. - Figures 5-8 give the photoelectron spectra for $\mathrm{N}_{2}, \mathrm{O}_{2}, \mathrm{CO}$, and $\mathrm{CO}_{2}$ using $\mathrm{Al} K_{\alpha} \mathrm{X}$-rays as a photon source. In each case we are examining the effects of excitation as the result of photoionization in the $K$ shell. The

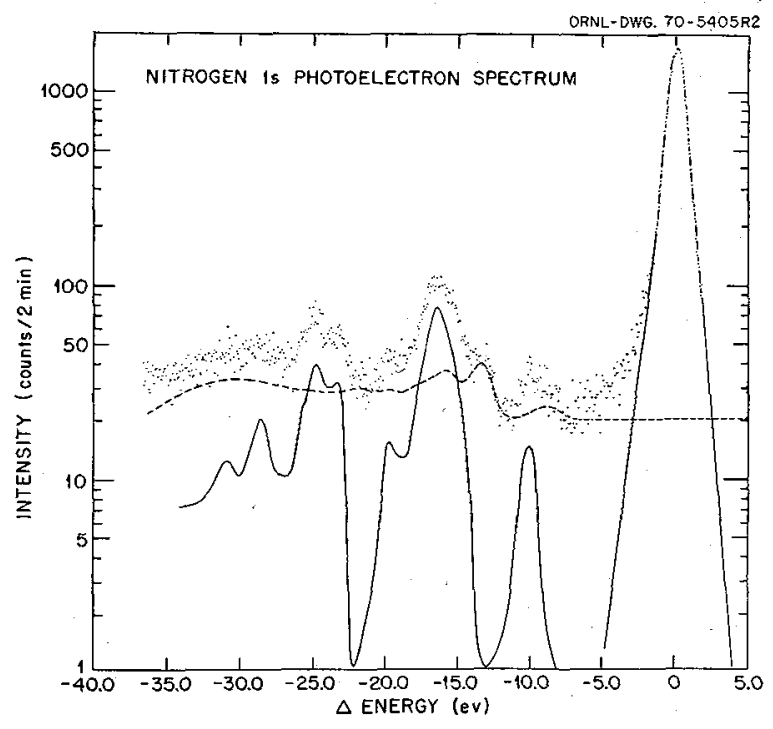

FIG. 5. - Photoelectron spectrum of $\mathrm{N}_{2}$ ionized in the $1 \mathrm{~s}$ shell by Al $K_{\alpha} \mathrm{X}$-rays. Energy given relative to principal peak whose kinetic energy is $1077 \mathrm{eV}$. Dots are data points. Dotted line gives contribution from background plus losses from inelastic scattering. Solid line is net spectrum. 


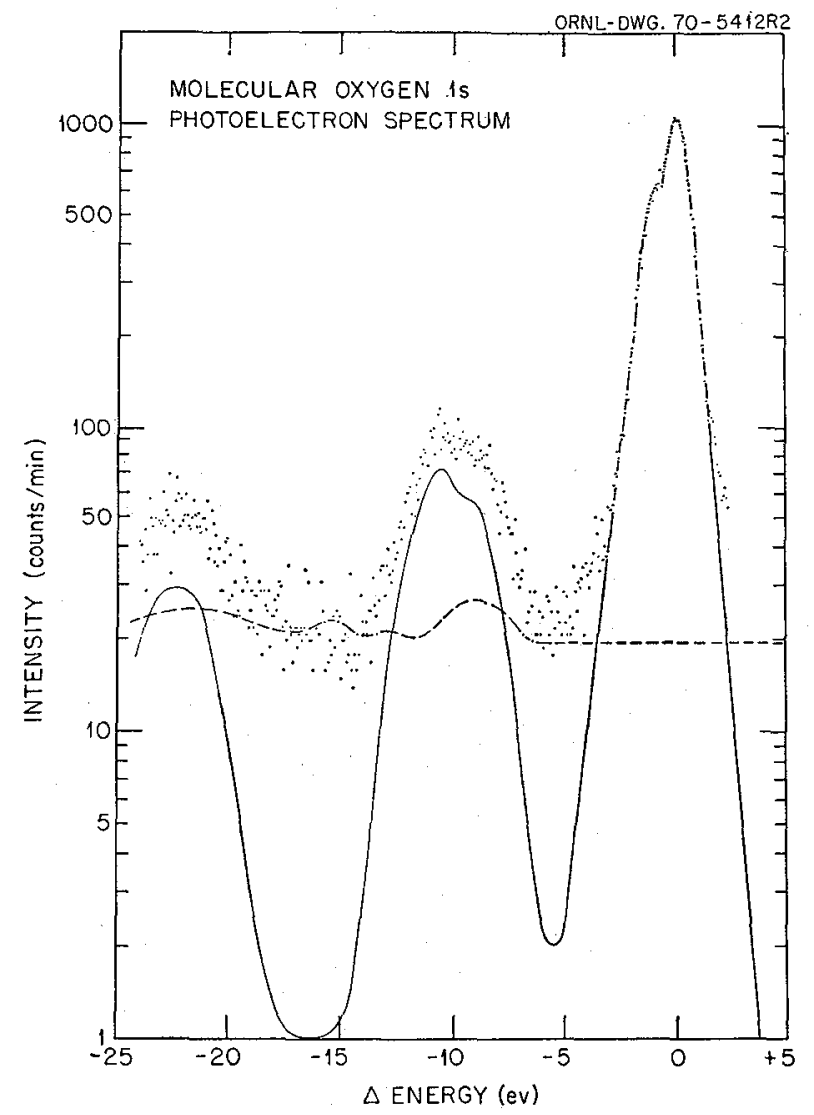

FIG. 6. - Photoelectron spectrum of $\mathrm{O}_{2}$ ionized in the $1 \mathrm{~s}$ shell by $\mathrm{Al} K_{\alpha} \mathrm{X}$-rays. Energy given relative to principal peak whose kinetic energy is $944 \mathrm{eV}$. Dots are data points. Dotted line gives contributions from background plus losses from inelastic scattering. Solid line is net spectrum.

doublet observed for the main photo peak in $\mathrm{O}_{2}$, however, arises from spin splitting [14]. The extent of monopole excitation or electron shake-up is considerable (from $\sim 10$ to $15 \%$ ) with observed contributions starting at from about 7.5 to $13 \mathrm{eV}$. One might expect as with atomic systems that selection rules for monopole transitions play an important role. Optical data is not available, however, for identification purposes and even crude estimates are difficult when the $K$ vacancy is localized on one of the atoms. In such a case the change in shielding cannot be considered simply a change in the central potential as far as the outer shell molecular orbitals are concerned. However, in the case of photoionization in the $1 \mathrm{~s}$ shell of $\mathrm{N}_{2}$ and in the $1 \mathrm{~s}$ shell associated with carbon for $\mathrm{CO}$, it is possible to make a good estimate of the energies involved by using data on $\mathrm{NO}^{+}$. The configuration of the valence electrons in $\mathrm{N}_{2}^{+}\left(1 \mathrm{~s}^{1}\right), \mathrm{CO}^{+}\left(1 \mathrm{~s}^{1}\right)$ and $\mathrm{NO}^{+}$(ground state) are identical viz., $\left(2 \mathrm{~s} \sigma^{*}\right)^{2}$ $\left(2 \mathrm{p} \pi^{\mathrm{b}}\right)^{4}\left(2 \mathrm{p} \sigma^{\mathrm{b}}\right)^{2}$. In addition, the valence electrons see nearly the same effective charge. That, is the effective nuclear charge felt by the valence electron is

$$
Z_{\text {eff }}=Z-\sigma
$$

where $Z$ is the nuclear charge and $\sigma$ is the shielding

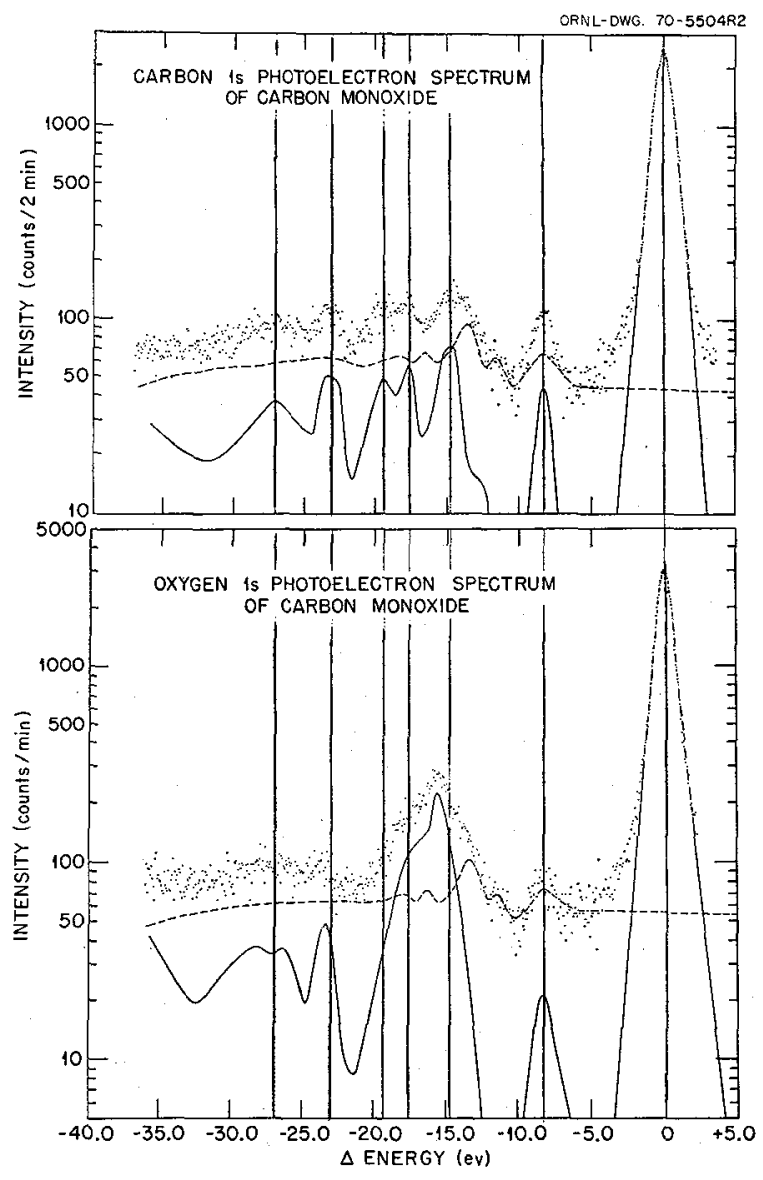

FIG. 7. - Photoelectron spectra of $\mathrm{CO}$ ionized in the $(a)$ is shell of carbon and $(b)$ in the $1 \mathrm{~s}$ shell of oxygen by $\mathrm{Al} K_{\alpha} \mathrm{X}$-rays. Energy given relative to principal peak whose kinetic energy is $1191 \mathrm{eV}$ for carbon and $944 \mathrm{eV}$ for oxygen. Dots are data points. Dotted line gives contribution from background plus losses from inelastic scattering. Solid lines are the net spectra. Vertical lines are drawn to help comparison of the peak values in the two spectra.

given by the electrons in the $1 \mathrm{~s}$ shell, which is slightly less than unity per electron. Thus, $Z_{\text {eff }}$ for the nitrogen atom with two $1 \mathrm{~s}$ electrons $\approx Z_{\text {eff }}$ for carbon with one $1 \mathrm{~s}$ electron and $Z_{\text {eff }}$ for oxygen with two $1 \mathrm{~s}$ electrons $\approx Z_{\text {eff }}$ for nitrogen with one $1 \mathrm{~s}$ electron.

The ground state of $\mathrm{NO}^{+}$is ${ }^{1} \Sigma^{+}$and in accordance with monopole excitation one would expect the final state to also be ${ }^{1} \Sigma^{+}$. From an analysis based on Gilmore [24] it would appear that the energy needed to excite an electron from the $2 \mathrm{p} \pi^{\mathrm{b}}$ orbital to the predissociated $2 \mathrm{p} \pi^{* 1} \Sigma^{+}$state is approximately 15 volts. Neglecting effects of spin coupling with unfilled $1 \mathrm{~s}$ shells, one would expect this energy to correspond to one of the peaks seen in the photoelectron spectra of $\mathrm{N}_{2}$ and $\mathrm{CO}$ (cf. Fig. 5 and 7). The most intense peak due to excitation does seem to appear in this energy region. The fact that it is the most intense is consistent with the fact that the $2 \mathrm{p} \pi^{\mathrm{b}}$ orbital contains 4 electrons and is thus the most populous of the valence shells.

Also, from the above arguments on the similarity 


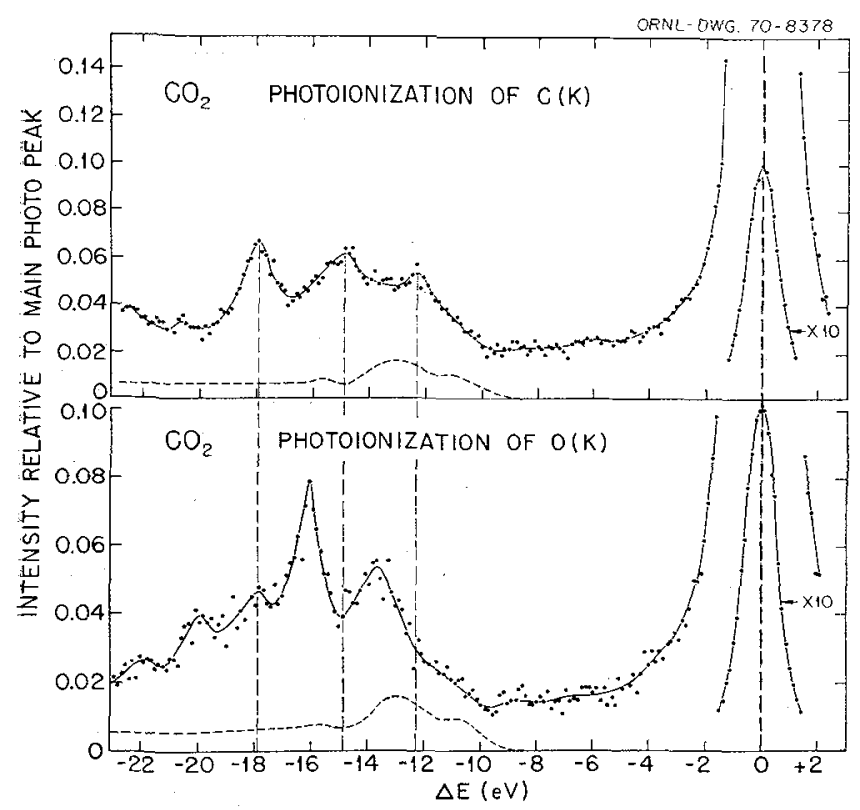

FIG. 8. - Photoelectron spectra of $\mathrm{CO}_{2}$ ionized in the $(a)$ $1 \mathrm{~s}$ shell of carbon and $(b)$ in the $1 \mathrm{~s}$ shell of oxygen by Al $K_{\alpha}$ $\mathrm{X}$-rays. Energy given relative to principal peak whose kinetic energy is $1189 \mathrm{eV}$ for carbon and $946 \mathrm{eV}$ for oxygen. Dots are data points. Dotted lines gives contribution from inelastic scattering. Vertical lines are drawn to help comparison of the peak values in the two spectra.

of $\mathrm{N}_{2}^{+}\left(1 \mathrm{~s}^{1}\right)$ and $\mathrm{CO}^{+}\left(\mathrm{C}_{1} \mathrm{~s}^{1}\right)$ one might expect that that the photoelectron spectra in figures 5 and 7 to be similar as far as the energy separation between the peaks corresponding to monopole excitation is concerned. The spectra do show such similarity. However, the relative intensities are different, but since the transition rates depend on the wave functions for the neutral molecules, these differences should be expected.

In $\mathrm{CO}$ and $\mathrm{CO}_{2}$ we have studied the effects of photoionization in the $K$ shell of both the oxygen and carbon. The spectra are easy to separate since the $K$ binding energy of carbon is about $280 \mathrm{eV}$ and that for oxygen $530 \mathrm{eV}$. It is interesting to note whether the energy and relative population of the excited states are the same or different depending on which atom the initial inner shell vacancy is found. In general, figures 7 and 8 show more differences than similarities although for $\mathrm{CO}$ a number of peaks corresponding to different excited states are approximately the same energy in both the carbon and oxygen studies.

A better understanding of the problem of inner shell vacancies in molecules than is afforded by the qualitative discussion given above awaits solutions of ab initio calculations on small molecules containing an inner shell vacancy. We hope to undertake such calculations in the near future.

One might also expect monopole excitation when photoionization occurs in the outer shells of molecules. Siegbahn [14] has indeed reported such a line in the photoionization of $\mathrm{N}_{2}$ using soft X-rays. The study of photoelectron spectra by use of the $21.22 \mathrm{eV}$ He with its inherent high resolution has been quite extensive but to our knowledge none of the spectra have thus far been discussed in terms of monopole excitation. In general 21 volts is insufficient to give rise to such states, but if the ionization potential for the least bound molecular orbital is about $10 \mathrm{eV}$ and the energy for excitation is less than about $10 \mathrm{eV}$ (which is quite reasonable as witness the states observed when $K$ photoionization occur) spectra could occur at kinetic energies of a couple of volts or less. In any case, as more data are taken with the He II resonance line $(40.8 \mathrm{eV})$ observations of simultaneous photoionization and excitation in molecules will surely be observed, and such studies will have the advantage of high resolution (20-40 millivolts).

Conclusion. - When photoionization occurs it can be accompanied by simultaneous excitation. This process can be studied by photoelectron spectroscopy, for when it occurs satellite lines appear on the low energy side of the main photo-peak. This paper has presented experimental results observed with a high resolution electron spectrometer on helium, neon, argon, $\mathrm{N}_{2}, \mathrm{O}_{2}, \mathrm{CO}$ and $\mathrm{CO}_{2}$.

The basic understanding of the problem seems well founded for atomic systems. When photoionization occurs in an inner shell, an electron in an outer shell orbital can be excited by the sudden change of the initial potential due to the loss of a shielding electron. The transitions are governed by monopole transition rules, and transition probabilities can be obtained in good agreement with experiment using Hartree-Fock single electron wave functions. When photoionization occurs in the outer shell, another electron in an orbital with the same principal quantum number can also undergo monopole excitation. The probability is, however, greatly underestimated by single-electron wave functions; but when electron correlation is explicitly included in the wave function for the initial states (as has been done in the case of helium) agreement with experiment is good.

It has been demonstrated that extensive excitation also occurs simultaneously with photoionization in the case of molecules, but a complete understanding of its nature is still in its infancy.

\section{References}

[1] Krause (M. O.), Vestal (M. L.), Johnston (W. H.) and Carlson (T. A.), Phys. Rev., 1964, 133, A 385.

[2] Carlson (T. A.) and Krause (M. O.), Phys. Rev., 1965,137, A1655.
[3] Carlson (T. A.) and Krause (M. O.), Phys. Rev., 1965,140, A 1057.

[4] Carlson (T. A.), Phys. Rev., 1967, 156, 142. 
[5] Krause (M. O.) and Carlson (T. A.), Phys. Rev., $1967,158,18$.

[6] Krause (M. O.), Carlson (T. A.), and Dismukes (R. D.), Phys. Rev., 1968, 170, 37.

[7] Utriainen (J.), Linkoaho (M.), Rantavuori (E.), ÅBerG (T.) and GraefFe (G.), Z. Naturforschung, 1968, 23a, 1178 .

[8] Krause (M. O.), Stevie (F. A.), Lewis (L. J.), CarlSON (T. A.) and Moddeman (W. E.), Phys. Letters, 1970, 31A, 81.

[9] Carlson (T. A.), Nestor (C. W.Jr. ), TuCker (T. C.) and MaLiK (F. B.), Phys. Rev., 1968, 169, 27.

[10] Áberg (T.), Phys. Rev., 1967, 156, 35.

[11] SAChenko (V. P.) and Demekhin (V. F.), Zh. Eksperim. i Teor. Fiz., 1965, 49, 765.

[12] Byron (F. W. Jr.), and JoachaIn (C. J.), Phys, Rev., 1967, 164, 1.

[13] Åberg (T.), Ann. Acad. Sci. Fenn AVI, 1969, 308, 1.

[14] Siegbahn (K.), Nordling (C.), Johansson (G.), Hedman (J.), Heden (P. F.), Hamrin (K.), Gelius (U.), Bergmark (T.), Werme (L. O.), ManNe (R.) and BAER (Y), ESCA Applied to Free Molecules (North Holland Pub. Co., Amsterdam, 1969).

[15] Pullen (B. P.), Carlson (T. A.) Moddeman (W. E.), Schweitzer (G. K.), Bull (W. E.), and Grimm (F. A.), J. of Chem. Phys., 1970, 53, 768.

[16] Bearden (J. A.), Rev. Mod. Phys., 1967, 39, 78.

[17] Bagus (P. S.) and Gelius (U.), to be published (Also see ref. 14 , p. 35).

[18] Calculated by C. Froese-Fischer and first reported in ref. 6.

[19] Samson (J. A. R.), Phys. Rev. Letters, 1969, 22, 693.

[20] Carlson (T. A.), Moddeman (W. E.) and Krause (M. O.), Phys. Rev. A, 1970, 1, 1406.

[21] Åberg (T.), Phys. Rev. A, 1970, 2, 1726.

[22] Moore (C. E.), "Atomic Energy Levels, Vol. I ) (N. B. S. 467, U. S. Govt. Printing Office, Washington, D. C. 1949).

[23] Brown (R. L.), Phys. Rev. A, 1970, 1, 341.

[24] Gilmore (F. R.), J. Quant. Spectros. Radiat. Transfer, $1965,5,369$.

\section{DISCUSSION}

MANNE. - The term monopole excitation relates to a particular model in which the processes are viewed, i. e. first ionization and then excitation. The alternative approach is to discard the one-electron approximation and consider the transitions as between atomic states described by the full $N$-electron wave functions including the expelled electrons. If one takes this view all transitions in photoelectron spectra are dipole transitions.

CARLSON. - Dr. Manne is quite correct. I should like to add that by making the "sudden approximation " in which one disregards the mechanism of the initial cause of the change in the central potential, a number of phenomena (eg. electrons shake-off in beta decay, photoionization, Auger processes, intermal conversion) can be related to a simple model which views the production of excited states as governed by monopole excitation.

HAENSEL. - In the optical excitation of $3 \mathrm{~s}$-Shell in $\operatorname{Ar}$ (Phys. Rev., 1968, 177, 136) and 2 s-shell of Ne (Phys. Rev., 1967, 155, 26), Madden, Ederer and Codling have observed window-and asymetric absorption-lines, which are due to interactions of the discrete final states of $3 \mathrm{~s}$ and $2 \mathrm{~s}$ transitions and transitions. of the outer most $p$ shell to continuum states. I wonder if there is any similar line shape observable in your electron spectra if $3 \mathrm{~s} \mathrm{Ar}$ or $2 \mathrm{~s} \mathrm{Ne}$ transition come into play.

CARLSON. - We have not observed any distortion of the line shapes. However, in using Al $K_{\alpha} \mathrm{X}$-rays, we have a net resolution of from 1.0 to $1.5 \mathrm{eV} \mathrm{f}$. w. h. m., which might tend to obscure such observations.

VAN DER WIEL. - The situation in the case of the experiment by the NBS-group is different, since they use photons of an energy resonant with the excitations considered, whereas Carlson uses photons of about $1 \mathrm{keV}$ above the excitation energy. 